\title{
Transient Expression of Secretory IgA In Planta is Optimal Using a Multi-Gene Vector and may be Further Enhanced by Improving Joining Chain Incorporation
}

Lotte B. Westerhof*, Ruud H. P. Wilbers, Debbie R. van Raaij, Christina Z. van Wijk, Aska Goverse, Jaap Bakker and Arjen Schots

Laboratory of Nematology, Plant Science Group, Wageningen University, Wageningen, Netherlands

Secretory $\lg A(\operatorname{sg} A)$ is a crucial antibody in host defense at mucosal surfaces. It is a promising antibody isotype in a variety of therapeutic settings such as passive vaccination and treatment of inflammatory disorders. However, heterologous production of this heteromultimeric protein complex is still suboptimal. The challenge is the coordinate expression of the four required polypeptides; the alpha heavy chain, the light chain, the joining chain, and part of the polymeric-lg-receptor called the secretory component, in a 4:4:1:1 ratio. We evaluated the transient expression of three slgAк variants, harboring the heavy chain isotype $\alpha 1$, $\alpha 2 \mathrm{~m} 1$, or $\alpha 2 \mathrm{~m} 2$, of the clinical antibody Ustekinumab in planta. Ustekinumab is directed against the p40 subunit that is shared by the pro-inflammatory cytokines interleukin (IL)-12 and IL-23. A slgA variant of this antibody may enable localized treatment of inflammatory bowel disease. Of the three different slgA variants we obtained the highest yield with slgA1 $\kappa$ reaching up to $373 \mu \mathrm{g}$ slgA/mg total soluble protein. The use of a multi-cassette vector containing all four expression cassettes was most efficient. However, not the expression strategy, but the incorporation of the joining chain turned out to be the limiting step for slgA production. Our data demonstrate that transient expression in planta is suitable for the economic production of heteromultimeric protein complexes such as slgA.

Keywords: secretory IgA, plant-based expression, Ustekinumab, $\mathbf{N}$-glycosylation, heteromultimeric, protein complex assembly, co-infiltration, multi-gene vector

\section{INTRODUCTION}

Secretory $\operatorname{Ig} \mathrm{A}(\mathrm{sg} \mathrm{A})$ is the predominant antibody type in mucosal secretions of the human body and plays a key role in the first line of defense against mucosal pathogens. While human serum IgA is primarily monomeric, B cells in mucosa-associated lymphoid tissues secrete IgA in a dimeric form through incorporation of the joining chain. Dimeric (d)IgA can bind the polymeric immunoglobulin receptor on the basolateral surface of epithelial cells where after the protein complex is transcytosed to the luminal side of the cell. Here the receptor is cleaved and a part of the receptor called the secretory component stays associated with the protein complex that is now referred to as secretory (s)IgA. Both dIgA and sIgA have immunological roles without development 
of inflammation. Mucosal antigens and/or pathogens can be bound by $\mathrm{d} / \mathrm{sIgA}$ just before, during (e.g., intracellular pathogens in the epithelial cells) or after transcytosis and are thereby excluded from the mucosal tissue. On the luminal side of epithelial cells glycans on the secretory component facilitate binding to the mucus thereby enabling clearance of sIgAantigen/pathogen complexes. This concept of antigen/pathogen binding and clearance that does not lead to inflammation is referred to as immune exclusion and is believed not only to play a role in combating mucosal pathogens, but also in controlling commensal bacteria. Inflammation does not develop because there is limited presence of FcaRI positive immune cells in the mucosal area and sIgA has reduced affinity for Fc $\alpha$ RI. In order to fulfill these roles the human body secretes 40-60 mg sIgA per kg body weight each day (Conley and Delacroix, 1987).

Two isotypes of IgA exist, IgA1 and IgA2 of which the latter occurs in two allotypes, $\operatorname{IgA} 2 \mathrm{~m} 1$ and $\operatorname{IgA} 2 \mathrm{~m} 2$. All three alpha heavy chains consist of one variable domain, three constant domains and an extended tailpiece allowing IgA to dimerise by incorporation of the joining chain. There are three major differences between these IgA variants. First, IgA1 has an extended hinge region. This extension is heavily $O$-glycosylated, which is suspected to play a conformational role (Narimatsu et al., 2010), allowing the binding of more distantly spaced antigens. Second, alpha heavy chains of isotypes 1 and $2 \mathrm{~m} 2$ are covalently linked to their light chains via a disulphide bridge. No such linkage exists in IgA2m1, which allows the formation of a intermolecular disulphide bridge between the two light chains. Third, all variants differ from each other in the number of $\mathrm{N}$-glycosylation sites they carry $(2,4$, and 5 for $\operatorname{IgA} 1, \operatorname{IgA} 2 \mathrm{~m} 1$ and $\operatorname{IgA} 2 \mathrm{~m} 2$, respectively). The ratio wherein sIgA isotypes are present depends on the mucosal area, which in turn is most likely the result of the presence of specific bacteria as the IgA1 hinge region is sensitive to bacterial proteases (Vaerman et al., 1968; Stoop et al., 1969; Delacroix et al., 1982; Kerr, 1990).

The use of recombinant sIgA in passive mucosal immunotherapy in humans and livestock has been suggested as a good alternative for antibiotics. Several in vivo studies demonstrated (s)IgA's potential to locally control mucosal pathogens, such as Mycobacterium tuberculosis in the lungs (Williams et al., 2004), Streptococcus mutans in the mouth (Ma et al., 1998), and Salmonella typhimurium, Vibrio cholera, and Cryptosporidium parvum in the gastrointestinal tract (Enriquez and Riggs, 1998). When sIgA would be directed against proinflammatory cytokines and administered to the gut, it may relieve symptoms and induce remission in patients suffering from inflammatory bowel diseases (IBDs). Current treatment of IBD often includes systemic application of anti-TNF- $\alpha$ or anti-IL-12/23 antibodies. However, application of anti-TNF- $\alpha$ antibodies has been associated with the onset of tuberculosis (Keane et al., 2001). Non-systemic cytokine neutralization may reduce such infection risks and other side effects. Because sIgA is stable in the gut, sIgA-based therapy could be localized. Luminal administration of an anti-TNF- $\alpha$ antibody was effective in mouse models of IBD (Bhol et al., 2013) and local drug administration has been suggested to be important for efficacy of IBD therapies (Neurath, 2014).
Plants are a promising production platform for pharmaceutical proteins. As eukaryotes they are able to correctly fold complex proteins and assemble protein complexes such as virus like particles (Chen and Lai, 2013) and antibodies (De Muynck et al., 2010). Compared to mammalian cells, plants are a more economic production platform, as they do not require expensive cell culture conditions. Furthermore, the $N$-glycosylation pathway has been engineered to facilitate expression of antibodies with humanized $\mathrm{N}$-glycans to allow effector functions (Bosch et al., 2013). Also, the engineering of mammalian mucin-type O-glycans has been achieved in plants (Castilho et al., 2012; Yang et al., 2012).

Plant-based expression of sIgA was achieved by stable transformation of the four individual genes required for $\operatorname{sgA}$ assembly followed by crossing the highest producers (Ma et al., 1995; Paul et al., 2014). A drawback of this strategy is that it is a lengthy and laborious process. Transient expression is much faster and almost always results in higher yields, as there is no constraint on expression imposed by the site of insertion in the plant genome. To achieve transient expression of more than one gene simultaneously, Agrobacterium cultures harboring vectors for the expression of the individual genes need to be co-infiltrated or a multi-cassette vector facilitating expression of all genes should be used. The risk with coinfiltration is that cells may not be transformed with all genes, but use of a multi-cassette vector may be impractical if expression of the individual proteins needs to be adjusted to reflect the stoichiometry of the protein complex. Transient expression of chicken sIgA was achieved by co-infiltration (Wieland et al., 2006) and human sIgA was transiently expressed with a multi-cassette vector system (Juarez et al., 2013; Paul et al., 2014). While above-mentioned studies achieved $\operatorname{sIgA}$ expression, they also showed the presence of a large proportion of monomeric IgA as well as other assembly intermediates. The presence of these assembly intermediates complicates downstream processing.

The objective of this study was to evaluate the plant-based expression and assembly of three sIgA variants of the clinical antibody Ustekinumab (CNTO1275) to unravel limitations in $\operatorname{sIgA}$ assembly. This antibody has specificity for the p40 subunit shared by interleukin-12 and interleukin-23 and may be used in IBD therapy. Changing the backbone from IgG to sIgA may enable local administration. First, we evaluated the transient expression of all individual genes required for sIgA assembly whereby the three alpha heavy chain types 1 , $2 \mathrm{~m} 1$, and $2 \mathrm{~m} 2$ were included. Next we compared co-expression with the use of multi-cassette vectors. The use of a multicassette vector including all genes increased sIgA expression threefold and decreased the presence of the intermediate $\operatorname{dIgA}$ compared to co-infiltration. However, sIgA expression may be further optimized, because we conclude that inefficient incorporation of the joining chain limits sIgA assembly. This maybe a consequence of inefficient $\mathrm{N}$-glycosylation of the IgA tailpiece and/or joining chain. Improved $\mathrm{N}$-glycosylation may be the key to enhance sIgA assembly en boost yield even further. 


\section{EXPERIMENTAL PROCEDURES}

\section{Construct Design}

GeneArt (Thermo Fisher, Bleiswijk, The Netherlands) synthesized all below-mentioned gene fragments except the constant region of human immunoglobulin alpha2m1 (AAB59396.1), which was amplified from the human transcriptome library MegaMan (Agilent Technologies, Middelburg, The Netherlands). Before gene synthesis, undesired restriction sites were removed from the sequences of the constant domains of human immunoglobulin alpha-1 (AAC82528.1) and kappa (AAA59000.1) chains, joining chain (AK312014.1) and secretory component (codons 1-764 of the polymeric immunoglobulin receptor; AAB23176.1). To obtain the sequence for the constant domains of human immunoglobulin alpha$2 \mathrm{~m} 2$ the sequence of human immunoglobulin alpha- $2 \mathrm{~m} 1$ was adapted (P93S, P102R, F279Y, D296E, L319M, V326I, and V335A) based on the amino acid sequence P01877 (Uniprot). The variable regions of the clinical antibody Ustekinumab (CNTO-1275) and the signal peptide of the Arabidopsis thaliana chitinase gene (AAM10081.1) were recoded from the amino acid sequence using codons preferred by Nicotiana benthamiana. For subsequent cloning and assembly of the alpha heavy chain genes, the gene fragments were flanked by the following restriction sites at the $5^{\prime}$ - and $3^{\prime}$-end: NcoI-EagI, EagI-NheI, NheI-KpnI, for the signal peptide, the heavy chain variable and alpha heavy chain constant regions, respectively. For subsequent cloning and assembly of the kappa chain, the gene fragments were flanked by the following restriction sites at the $5^{\prime}$ - and $3^{\prime}$-end: NcoI-EagI, EagI-BsiWI, BsiWI-KpnI for the signal peptide, the kappa chain variable and constant region, respectively. For subsequent cloning of the joining chain and secretory component the sequences were flanked by NcoI-KpnI at the $5^{\prime}$ - and $3^{\prime}$-end, respectively. None of the restriction sites used introduced extra amino acids except NcoI, which in some cases introduced an extra alanine after the start methionine. Genes were ligated into the shuttle vector pRAPa, a pRAP (or pUCAP35S) derivative (van Engelen et al., 1994) modified to include an AsiSI restriction site by introduction of the selfannealed oligo $5^{\prime}$ - AGCTGGCGATCGCC $-3^{\prime}$ into a HindIII linearized pRAP. In pRAPa all open reading frames are placed under the control of the $35 \mathrm{~S}$ promoter of the Cauliflower mosaic virus with duplicated enhancer (d35S) and the Agrobacterium tumefaciens nopaline synthase transcription terminator (Tnos). A $5^{\prime}$ leader sequence of the Alfalfa mosaic virus RNA 4 (AlMV) is also included between the promoter and gene to boost translation. From pRAPa the expression cassettes were digested with AscI and PacI, confirmed by sequencing, and ligated into the expression vector pHYG (Westerhof et al., 2012). Use of the restriction sites AscI and AsiSI allowed subsequent introduction of expression cassettes as AsiSI creates the same overhang as PacI (Figure 1B). Three multi-cassette vectors were generated; one that combined the alpha chain-1 and the light chain, one that combined the alpha chain-1, the light chain and the joining chain and one that combined the alpha chain-1, the light chain, the joining chain and the secretory component. Expression vectors were transformed to Agrobacterium tumefaciens strain MOG101 for transient plant expression.

\section{Transient Plant Transformation}

Agrobacterium clones were cultured overnight $(\mathrm{o} / \mathrm{n})$ at $28^{\circ} \mathrm{C}$ in LB medium ( $10 \mathrm{~g} / \mathrm{l}$ pepton $140,5 \mathrm{~g} / \mathrm{l}$ yeast extract, $10 \mathrm{~g} / \mathrm{l} \mathrm{NaCl}$ with $\mathrm{pH} 7.0)$ containing $50 \mu \mathrm{g} / \mathrm{ml}$ kanamycin and $20 \mu \mathrm{g} / \mathrm{ml}$ rifampicin. The optical density (OD) of the o/n cultures was measured at $600 \mathrm{~nm}$ and used to inoculate $50 \mathrm{ml}$ of $\mathrm{LB}$ medium containing $200 \mu \mathrm{M}$ acetosyringone and $50 \mu \mathrm{g} / \mathrm{ml}$ kanamycin with $x \mu \mathrm{l}$ of culture using the following formula: $x=80000 /\left(1028^{*} \mathrm{OD}\right)$. OD was measured again after $16 \mathrm{~h}$ and the bacterial cultures were centrifuged for $15 \mathrm{~min}$ at $2800 \times g$. The bacteria were resuspended in MMA infiltration medium (20 g/l sucrose, $5 \mathrm{~g} / \mathrm{l}$ MS-salts, $1.95 \mathrm{~g} / \mathrm{l} \mathrm{MES,} \mathrm{pH}$ 5.6) containing $200 \mu \mathrm{M}$ acetosyringone. For co-expression of genes Agrobacterium cultures harboring expression vectors for individual gene expression were mixed prior to infiltration or a multi-cassette vector was used. The final OD of each Agrobacterium culture in an infiltration mix was 0.5 unless indicated otherwise. The total OD of the infiltration mix was kept the same within an experiment by using an Agrobacterium culture harboring an empty vector if needed. The Tomato bushy stunt virus (TBSV) silencing inhibitor p19 was always co-expressed (Voinnet et al., 2003). After 1-2 h incubation of the infiltration mix at room temperature, the two youngest fully expanded leaves of 5-6 weeks old Nicotiana benthamiana plants were infiltrated completely. Infiltration was performed by injecting the Agrobacterium suspension into a Nicotiana benthamiana leaf at the abaxial side using a needleless $1 \mathrm{ml}$ syringe. Infiltrated plants were maintained in a controlled greenhouse compartment (UNIFARM, Wageningen) and infiltrated leaves were harvested at selected time points.

\section{Total Soluble Protein Extraction}

Leaf disks were taken from fully infiltrated leaves and immediately snap-frozen. Plant material was ground first in liquid nitrogen and then in $2 \mathrm{ml}$ ice-cold extraction buffer [50 mM phosphate-buffered saline (PBS) $\mathrm{pH}=7.4,100 \mathrm{mM}$ $\mathrm{NaCl}, 10 \mathrm{mM}$ ethylenediaminetetraacetic acid (EDTA), 0.1\% $\mathrm{v} / \mathrm{v}$ Tween-20, 2\% w/v immobilized polyvinylpolypyrrolidone (PVPP)] per g fresh weight using a TissueLyser II (Qiagen, Venlo, The Netherlands). Crude extract was clarified by centrifugation at $16.000 \times g$ for $5 \mathrm{~min}$ at $4^{\circ} \mathrm{C}$.

\section{IgA and slgA Quantification}

IgA and sIgA concentrations in crude extracts were determined by sandwich ELISA. ELISA plates (Greiner Bio One; Alphen aan den Rijn, The Netherlands) were coated $\mathrm{o} / \mathrm{n}$ at $4^{\circ} \mathrm{C}$ in a moist environment with goat polyclonal anti-human kappa antibody (Sigma-Aldrich; Zwijndrecht, The Netherlands) in coating buffer (eBioscience, Vienna, Austria). After this and each following step the plate was washed five times with $30 \mathrm{~s}$ intervals in PBST ( $1 \mathrm{x}$ PBS, $0.05 \%$ Tween-20) using an automatic plate washer model 1575 (BioRad; Veenendaal, The Netherlands). The plate was blocked with assay diluent 
(eBioscience) for $1 \mathrm{~h}$ at room temperature. Samples and a standard line were loaded in serial dilutions and incubated for $1 \mathrm{~h}$ at room temperature. For IgA determination recombinant human IgA1к (InvivoGen; Toulouse, France) was used as a standard in a twofold dilution series from 100 to $0.31 \mathrm{ng} / \mathrm{ml}$ in assay diluent. For sIgA determination colostrum purified sIgA (Sigma-Aldrich) was used as a standard in a twofold dilution series from 1000 to $3.1 \mathrm{ng} / \mathrm{ml}$ in assay diluent. Hereafter a HRP-conjugated goat polyclonal antibody directed against the constant domains of human IgA (Sigma-Aldrich) or a biotinylated goat polyclonal antibody directed against the human secretory component (Sigma-Aldrich) was used for detection of IgA and sIgA, respectively. Avidin-HRP conjugate (eBioscience) was used to bind the biotin of the antisecretory component antibody. 3,3',5,5'-Tetramethylbenzidine (TMB) substrate (eBioscience) was added and coloring reaction was stopped using $0.18 \mathrm{M}$ sulphuric acid after 1-30 min. OD read outs were performed using the model 680 microplate reader (BioRad) at $450 \mathrm{~nm}$ with correction filter of $690 \mathrm{~nm}$. For sample comparison the total soluble protein (TSP) concentration was determined using the BCA Protein Assay Kit (Pierce) according to supplier's protocol using bovine serum albumin (BSA) as a standard.

\section{Protein Analysis by Western Blot}

For western blot analysis clarified protein extracts were desalted using a Sephadex G25 (VWR International; Amsterdam, The Netherlands) column prior to BCA analysis. One microgram (unless otherwise indicated) of TSP was separated under reducing or non-reducing conditions by SDS-PAGE on in house made 6 or $12 \%$ Bis-Tris gels. Recombinant $\operatorname{IgA} 1 \kappa$ (InvivoGen) and/or colostrum purified sIgA (Sigma-Aldrich) or recombinant joining chain (Sino Biological; Cologne, Germany) were used as controls. Proteins were transferred to an
Invitrolon $^{\mathrm{TM}}$ PVDF membrane (Invitrogen) by a wet blotting procedure (Life technologies; Bleiswijk, The Netherlands). Thereafter the membrane was blocked in PBST-BL (PBS containing $0.1 \% \mathrm{v} / \mathrm{v}$ Tween-20 and $5 \% \mathrm{w} / \mathrm{v}$ non-fat dry milk powder) for $1 \mathrm{~h}$ at room temperature, followed by overnight incubation with a goat anti-human kappa (Sigma-Aldrich), goat anti-human immunoglobulin alpha (Sigma-Aldrich), goat anti-joining chain (Nordic Immunological Laboratories; Tilburg, The Netherlands) or goat anti-secretory component (Sigma-Aldrich) antibody. The membrane was washed five times in PBST (PBS containing 0.1\% v/v Tween-20). There after a HRP-conjugated anti-goat IgG antibody (Jackson ImmunoResearch; Suffolk, UK) was incubated with the membrane where after washing steps were repeated. The SuperSignal West Dura substrate (Thermo Fisher Scientific; Etten-Leur, The Netherlands) was used for visualization. Pictures were taken using a G:BOX Chemi System device (SynGene; Cambridge, UK).

\section{RESULTS}

\section{Expression of Individual Components Required for sIgA Assembly}

In order to achieve in vivo assembly of a heteromultimeric protein complex all required genes should be expressed simultaneously in the same cell and preferably at the right stoichiometry. The sIgA protein complex comprises four proteins. An overview of the individual proteins and the protein complexes IgA, dimeric (d)IgA and sIgA based on alpha heavy chain isotype 1 is given in Figure 1A. Expression cassettes and the combinations thereof to achieve expression of these protein complexes are given in Figure 1B. To evaluate the level and course of expression
A

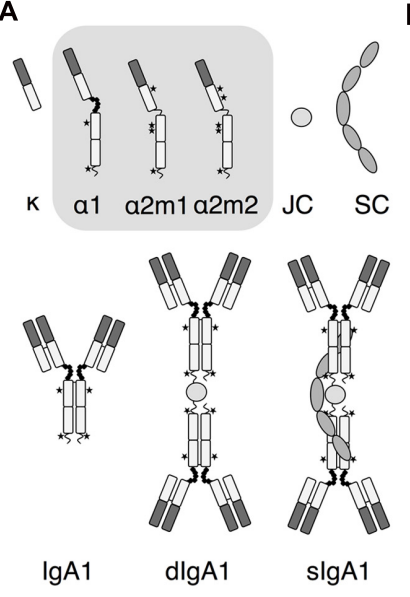

B

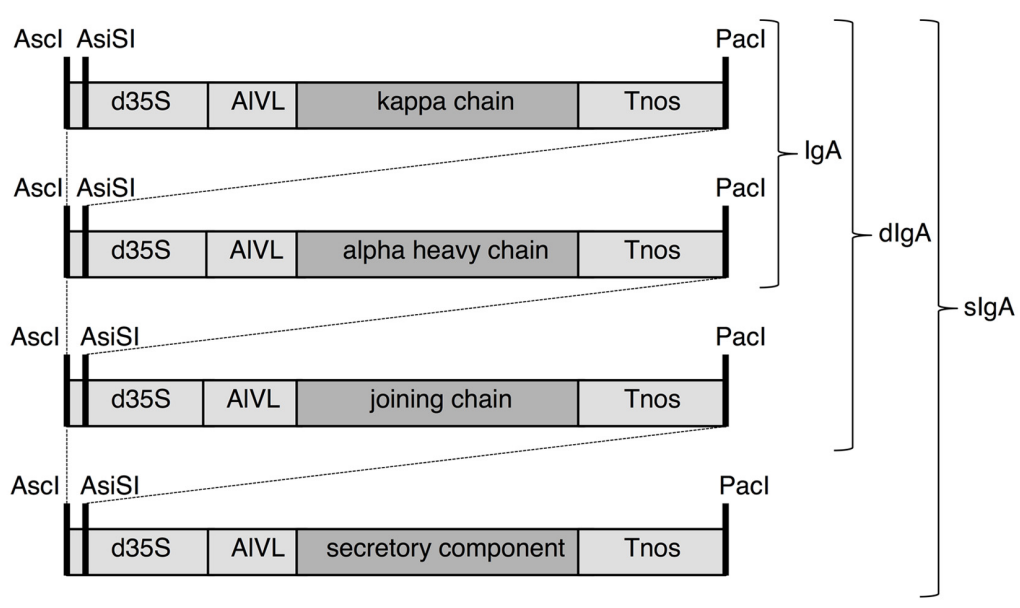

FIGURE 1 | Overview of sIgA assembly and expression. (A) Individual proteins, alpha heavy chain $1\left(\alpha^{1}\right), 2 \mathrm{~m} 1\left(\alpha^{2 m 1}\right)$, or $2 \mathrm{~m} 2\left(\alpha^{2 m 2}\right)$, kappa chain $(\kappa)$, joining

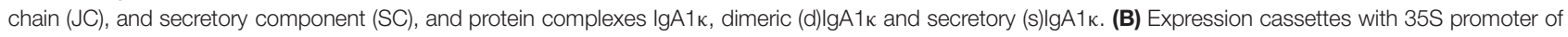
the Cauliflower mosaic virus with duplicated enhancer (d35S), Agrobacterium tumefaciens nopaline synthase transcription terminator (Tnos), translational leader sequence of the Alfalfa mosaic virus RNA 4 (AIVL) and Ascl, AsiSI and Pacl restriction sites are indicated. Co-expression is required for IgA, dlgA, and slgA assembly as indicated by the accolades. 
of the individual genes we first expressed them individually and monitored expression over time [3, 6, and 9 days post infiltration (dpi)] using western blot analysis (Figure 2). For the alpha heavy chain the isotypes $1,2 \mathrm{~m} 1$, and $2 \mathrm{~m} 2$ were evaluated. Upon expression of the alpha heavy chains and the kappa light chain bands were detected at the expected sizes that are assumed to represent the intact proteins. Furthermore, for all heavy chains several bands $>100 \mathrm{kDa}$ and a few faint bands $<50 \mathrm{kDa}$ were observed, which most likely represent multimers and products of proteolytic degradation, respectively. Upon expression of the secretory component a band was detected that migrates $\sim 10 \mathrm{kDa}$ lower compared to the secretory component of the sIgA control. This may be explained by a difference in the number and/or type of $N$-glycans received by the secretory component when expressed in plants. The secretory component has seven confirmed glycosylation sites, but these may not (all) be glycosylated in plants. Furthermore, the most common $N$-glycans of plant-secreted proteins are $0.7-1.1 \mathrm{kDa}$ smaller than most typical $N$-glycans found on human secretory component (Royle et al., 2003) which could already account for $\sim 10 \mathrm{kDa}$ difference in protein size. Upon expression of the joining chain many bands were detected, but all migrate higher as expected for a single joining chain (15.6-17.1 kDa, depending on glycosylation) and most of them likely represent dimers/multimers. Also the E. coli produced recombinant joining chain (expected size $\sim 17 \mathrm{kDa}$ ) displays aberrant migration behavior and migrates around $20 \mathrm{kDa}$.

The course of expression of the individual proteins is similar and peaks at $6 \mathrm{dpi}$, except for alpha heavy chain $2 \mathrm{~m} 2$, which peaks at 9 dpi. However, yields of the individual proteins vary. By comparing the band intensity of each individual protein to the recombinant controls we estimate yields between 1 and $5 \mu \mathrm{g} / \mathrm{mg}$ TSP for the alpha heavy chains, between 5 and $20 \mu \mathrm{g} / \mathrm{mg}$ TSP for the joining chain and between 50 and $200 \mu \mathrm{g} / \mathrm{mg}$ TSP for the kappa chain and the secretory component at dpi 6. Yield estimation of the heavy chains and joining chain is solely based on the intact monomeric proteins and is most likely underestimated due to the presence of multimers. Nonetheless, as the stoichiometric ratio between the heavy chain, the kappa chain, the joining chain and the secretory component is $4: 4: 1: 1$, we assume heavy chain expression to be the limiting factor for sIgA assembly if stabilization of individual proteins upon co-expression would not occur.

\section{A Multi-Cassette Vector is Most Efficient for Transient Expression of slgA}

Co-expression in transient transformation can be achieved in two ways, either by co-infiltration of Agrobacterium cultures harboring a vector for each individual gene or by using a multicassette vector facilitating expression of all genes. Which strategy

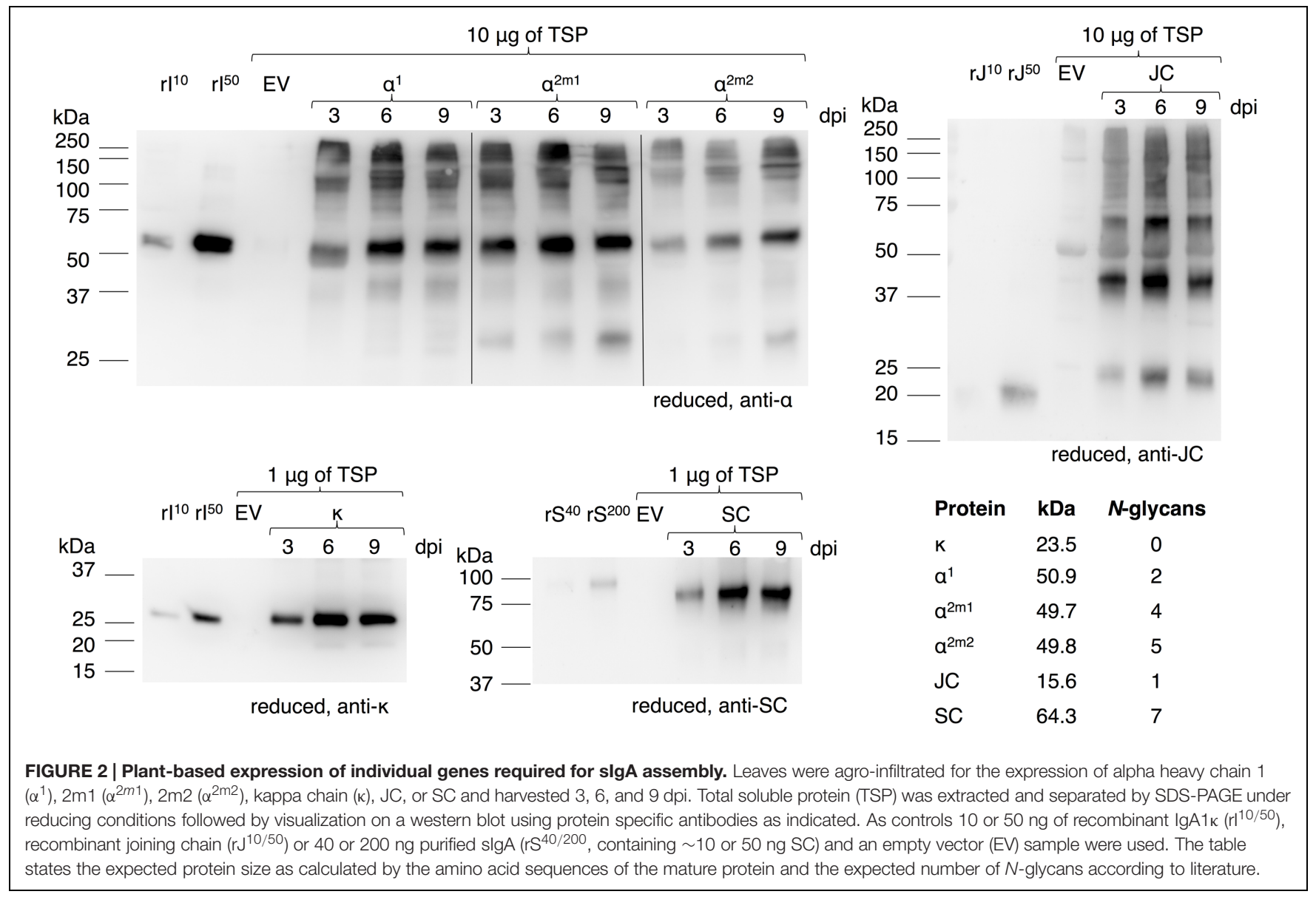


would lead to the best co-ordinated expression is unclear. With co-infiltration it is possible that not all cells are transformed with all expression cassettes. The use of a multi-cassette vector would ensure that a transformed cell receives all genes, however, transformation may be less efficient due to the larger size of the T-DNA. Therefore we used both strategies and combinations thereof to express all genes needed for sIgA complex formation. We successfully constructed several multi-cassette vectors for

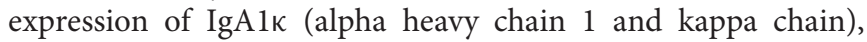

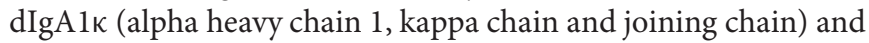
sIgA $1 \kappa$ (all four genes) whereby all genes are under control of the same promoter and terminator (Figure 1B). Subsequently, sIgA was expressed by co-infiltration of all genes individually (4vector system), co-infiltration of the secretory component and the

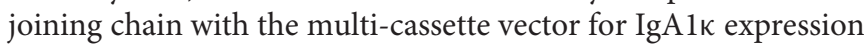
(3-vector system), co-infiltration of the secretory component and the multi-cassette vector for $\operatorname{dIgA} 1 \kappa$ expression (2-vector system) and the infiltration of the multi-cassette vector for $\operatorname{sigA} 1 \kappa$ expression (1-vector system; Figure 3B). Figure $\mathbf{3 A}$ shows the average sIgA yield of three biological replicates as determined by ELISA. To correct for the lower OD of the final Agrobacterium infiltration mix of the 3-, 2-, and 1-vector systems compared to the 4-vector system, an Agrobacterium culture carrying an empty vector (EV) was used to increase the OD (gray bars) or the concentration of the Agrobacterium carrying the multicassette vector was increased (dark gray bars). In both situations the yield is similar between the 4-, 3-, and 2-vector systems. Surprisingly, however, the use of the 1-vector system increases yield twofold to threefold. In the situation where the OD of the Agrobacterium cultures were supplemented with EV culture (gray bars), the twofold yield increase may be explained by an increased number of cells that receives all genes. In the case where we compensated the OD of the Agrobacterium cultures with cultures harboring the multi-cassette vector (dark gray bars) we were able to enhance sIgA yield 1.6-fold further. Use of a higher OD of an Agrobacterium culture often increases yield, as more T-DNA copies are transferred to the plant cell.
Noteworthy is that despite the fact that in our 1-vector system the same promoter and terminator sequences were used to facilitate expression of all genes, loss of vector parts or loss of sIgA expression upon plant transformation was never observed. We therefore assume that our multi-cassette expression vectors are stable and recombination did not occur. These data suggest that the use of a multi-cassette vector is the most efficient strategy for transient expression of heteromultimeric protein complexes.

\section{Co-Expression of Alpha Heavy Chain and Kappa Chain Stabilizes Both Proteins}

To determine the limiting factor in sIgA assembly we first evaluated the efficiency of IgA assembly in the absence of the joining chain and secretory component. Thereto, we co-expressed the alpha heavy chains and the kappa chain using the dual-cassette expression vectors for all three $\operatorname{IgA}$ variants and compared it with the individual expression of the alpha heavy chains and kappa chains. Leaf extracts were analyzed by western blot under reducing and non-reducing conditions (Figure 4). Visualization of the alpha heavy chains and kappa chain under reducing conditions demonstrated that all proteins accumulate to a higher level upon coexpression. As the accumulation of the alpha heavy chains increases upon co-expression with the light chain the presence of a degradation product just above $25 \mathrm{kDa}$ becomes clear. Considering the size of this degradation product it is most likely the result of cleavage in the hinge region. All three alpha heavy chains seem sensitive to proteolysis of the hinge region.

While analyzing co-expression of alpha heavy chains with kappa light chain under non-reducing conditions, a band around the expected size for $\operatorname{IgA}(\sim 150 \mathrm{kDa})$ was detected on blots either treated with anti-alpha heavy chain or antikappa chain specific antibodies. We therefore assumed that this band represents intact IgA complex. Next to the $150 \mathrm{kDa}$ band, several bands migrating $>250 \mathrm{kDa}$ were detected. As these bands were also seen in the recombinant IgA control

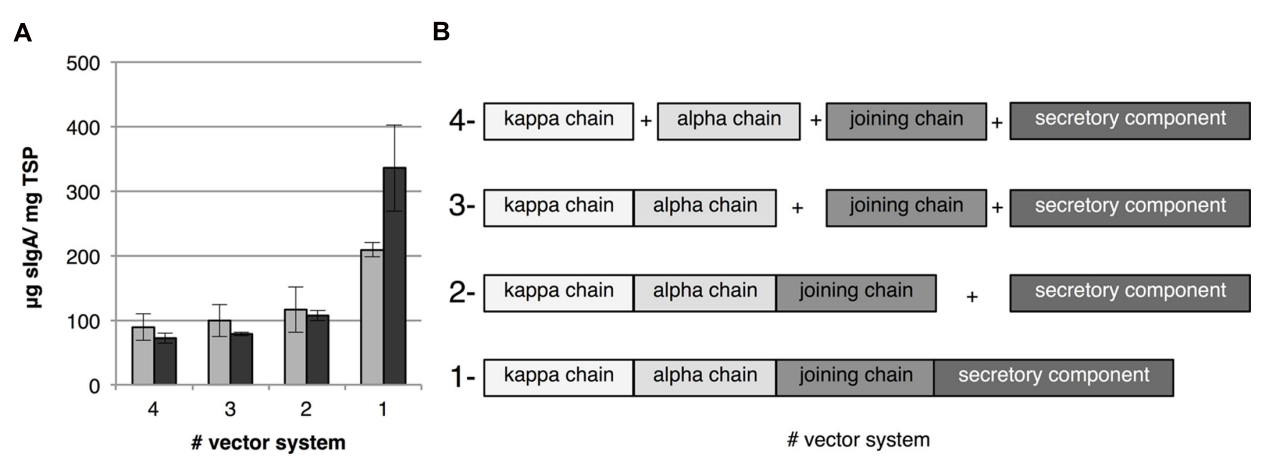

FIGURE 3 | slgA yield upon transient transformation using co-infiltration and multi-cassette vectors. (A) Average slgA yield 6 dpi of three biological replicates in leaf extracts when co-infiltrating all genes individually, co-infiltrating the secretory component and joining chain with the multi-cassette vector for lgA $1 \kappa$ expression, co-infiltrating the secretory component and the multi-cassette vector for dlgA expression and the infiltration of the multi-cassette vector for slgA expression indicated as the 4-, 3-, 2-, and 1-vector system, respectively. The final OD of the infiltration mix was set to 2.5 using either an Agrobacterium culture carrying an EV (light gray bars) or the OD of the Agrobacterium culture carrying the multi-cassette vector was increased (dark gray bars). Error bars indicate standard error. (B) A schematic overview of the expression cassettes in the 4-, 3-, 2-, and 1-vector systems. 

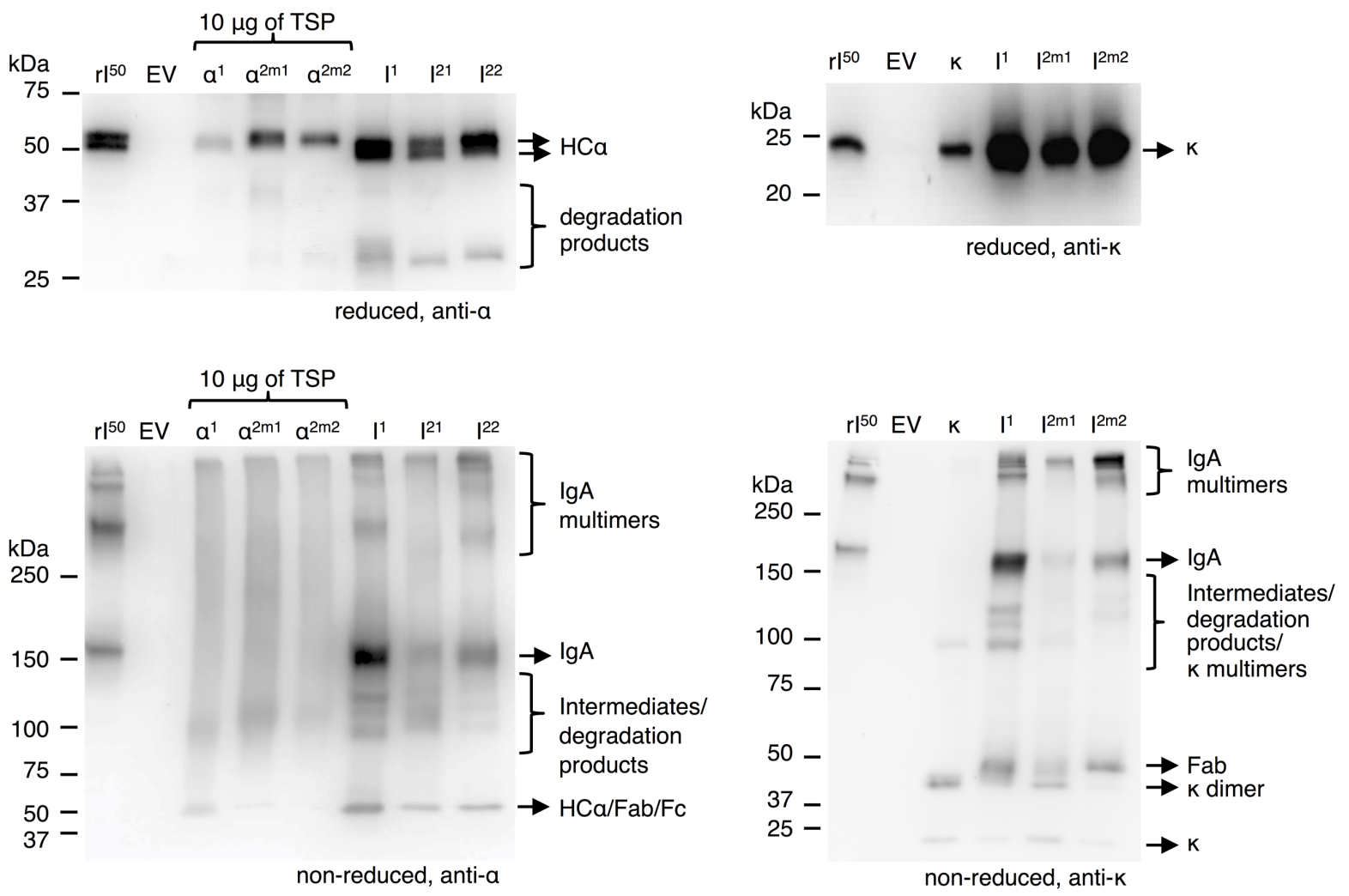

FIGURE 4 | IgA assembly using multi-cassette expression vectors. Leaves were agro-infiltrated for the expression of alpha heavy chain 1 ( $\left.\alpha^{1}\right)$, $2 \mathrm{~m} 1$ ( $\alpha^{2 m 1}$ ), $2 \mathrm{~m} 2\left(\alpha^{2 \mathrm{~m} 2}\right)$, and kappa chain ( $\left.\mathrm{\kappa}\right)$ individually or co-expressed $(\mathrm{I})$ using a multi-cassette vector and harvested 6 dpi. TSPs were extracted and $1 \mu \mathrm{g}$, unless stated otherwise, was separated by SDS-PAGE under reducing conditions followed by visualization using protein specific antibodies as indicated. As controls 50 ng of recombinant $\lg A 1 \kappa\left(r r^{50}\right)$ and an EV sample were used.

we assume they represent dimers/multimers of IgA. Also, several bands $<150 \mathrm{kDa}$ were detected, which may represent assembly intermediates, degradation products and/or individual polypeptide chains. Proteolytic cleavage in the hinge region results in Fc and Fab fragments of the same size as an intact alpha heavy chain $(\sim 50 \mathrm{kDa})$. A band of $50 \mathrm{kDa}$ is clearly detected on both alpha heavy chain and the kappa chain specific blots and therefore most likely represents Fab fragments. Two bands only detected on the kappa chain specific blot just below 25 and $50 \mathrm{kDa}$ most likely represent un-associated monomeric and dimeric kappa chains. Assuming no un-associated alpha heavy chain is present, we conclude that accumulation of the alpha heavy chain is the limiting factor for IgA yield, despite the fact that the alpha heavy chains stabilize upon co-expression with the kappa chain.

\section{IgA Dimerization is the Limiting Step in slgA Assembly}

Next we evaluated the efficiency of sIgA assembly. We used the multi-cassette vectors to express $\operatorname{IgA}, \operatorname{dIgA}$, and $\operatorname{sigA}$ (Figure 3B) with all three alpha heavy chain variants. Leaf extracts were analyzed on western blot under non-reducing conditions (Figure 5). Upon $\operatorname{dIgA} 1, \operatorname{dIgA} 2 \mathrm{~m} 1$, and $\operatorname{dIgA} 2 \mathrm{~m} 2$ expression two bands around 300 and $>420 \mathrm{kDa}$ were detected on the joining chain specific blot (third panel from the top). These bands can also be seen on the alpha heavy chain and kappa chain specific blots (first and second panel, respectively). We assume that these bands represent dIgA and multimerized (d)IgA. The $150 \mathrm{kDa}$ band representing monomeric IgA in the alpha heavy chain and kappa chain specific blots is still present upon coexpression of the joining chain. This implies that dimerization of $\operatorname{IgA}$ is not $100 \%$ efficient or that the expression of the joining chain is limiting. The presence of free joining chains was not detected.

Upon sIgA1 expression monomeric IgA $(\sim 150 \mathrm{kDa})$ is still detected on the alpha heavy chain and kappa chain specific blots. Next to that, bands of $<100, \sim 220, \sim 400$ and $>420 \mathrm{kDa}$ were detected on the secretory component specific blot (bottom panel). Because the band $<100 \mathrm{kDa}$ was only detected on the secretory component specific blot, this band most likely represents free secretory component. The $220 \mathrm{kDa}$ band was also detected on the alpha heavy chain and kappa chain specific blot, but not on the joining chain specific blot and therefore most likely represents secretory component associated with monomeric IgA. Both the $\sim 400$ and $>420 \mathrm{kDa}$ bands were also detected on the alpha heavy chain, kappa chain and joining chain blot and therefore must represent $\operatorname{sIgA}$ and multimers of $(\mathrm{d} / \mathrm{s}) \operatorname{IgA}$. Upon $\operatorname{sg} \mathrm{A} 2 \mathrm{~m} 1$ and $\operatorname{sigA} 2 \mathrm{~m} 2$ expression only the band $<100 \mathrm{kDa}$ representing 

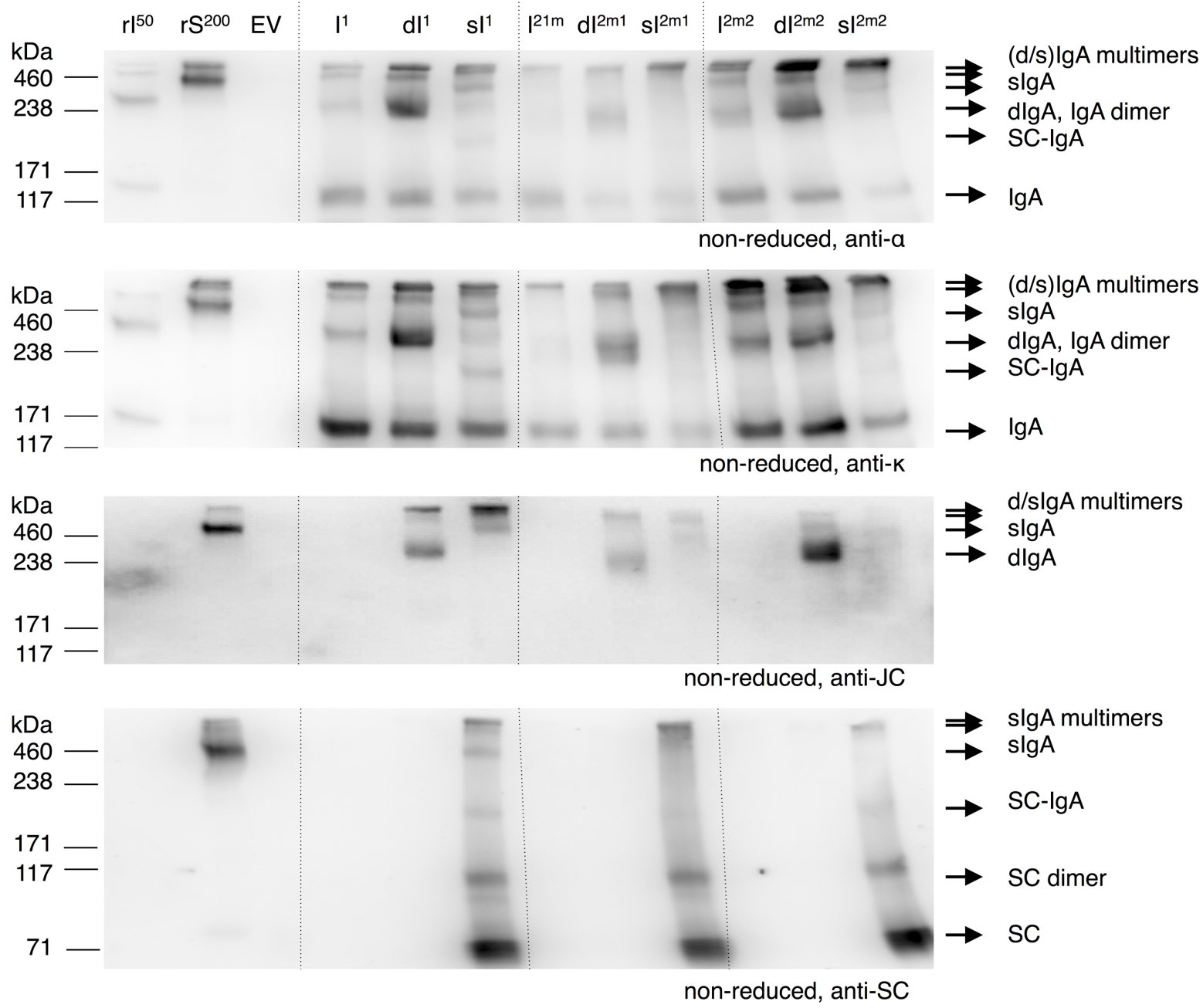

FIGURE 5 | slgA assembly using multi-cassette expression vectors. Leaves were agro-infiltrated for the expression of IgA1 $\left(I^{1}\right)$, $2 \mathrm{~m} 1$ ( $\left.\left.\right|^{2 m 1}\right)$, or $2 \mathrm{~m} 2$ ( $\left({ }^{2 m} 2\right)$ together with joining chain (dl) and secretory component (sl) using multi-cassette vectors and harvested 6 dpi. TSPs were extracted and $1 \mu \mathrm{g}$ was separated by SDS-PAGE under reducing conditions followed by visualization using protein specific antibodies as indicated. As controls 50 ng of recombinant lgA1 $\kappa\left(\mathrm{rl}{ }^{50}\right.$ ) and 200 ng purified slgA ( $\left.\mathrm{SS}^{200}\right)$ and an EV sample were used.

free secretory component and the band $>420 \mathrm{kDa}$ representing multimeric sIgA was clearly distinguished. It may be that alpha heavy chains $2 \mathrm{~m} 1$ and $2 \mathrm{~m} 2$ are more inclined to multimerization. No dIgA was detected upon expression of all four genes using any of the alpha heavy chains. Apparently sIgA assembly is equally efficient for all three alpha heavy chains and the expression of the secretory component is not limiting. The latter can also be concluded by the ample presence of free secretory component in the sIgA samples.

Both IgA and sIgA yield were determined with a sandwich ELISA using an anti-light chain capture antibody and an antialpha chain or anti-secretory component detection antibody, respectively. Although we speak of IgA and sIgA yield, it should be stated that also $\operatorname{IgA}$ and $\operatorname{sg} \mathrm{A}$ intermediates may be detected. While yields of the $\operatorname{IgA}$ variants ranged between 10 and $40 \mu \mathrm{g} / \mathrm{mg}$ TSP, sIgA yield was at least ninefold higher for all variants (Figure 6). The higher sIgA yield, compared to IgA yield, may be explained by the fact that sIgA assembly prevents proteolytic degradation of IgA. However, sIgA yield may be somewhat overestimated due to the presence of monomeric IgA associated with the secretory component.

These western blotting and ELISA results show that dimerization of IgA is the limiting step for sIgA assembly. Improving dIgA assembly could further increase sIgA yield and reduce the presence of assembly intermediates thereby simplifying down-stream processing.

\section{Joining Chain Incorporation is the Limiting Factor for slgA Yield}

Next, we investigated whether joining chain expression is the limiting factor for sIgA assembly. Thereto, we attempted to increase the expression of the joining chain by increasing the OD of the Agrobacterium culture harboring the vector for joining 

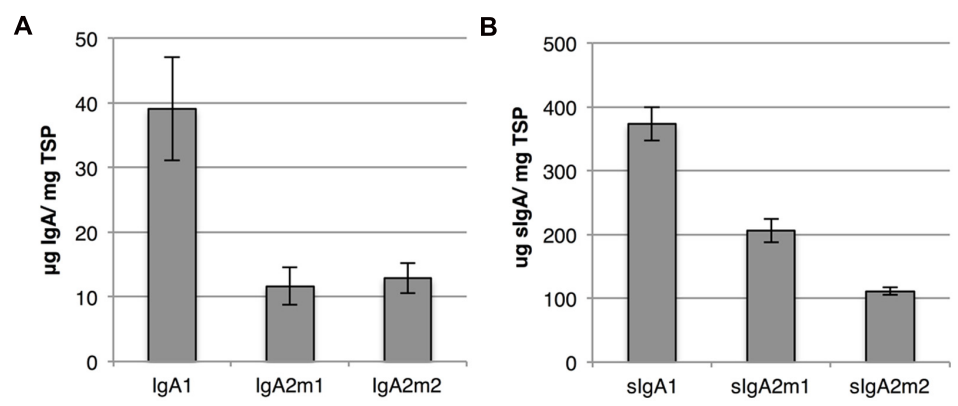

FIGURE 6 | IgA and sIgA yield using multi-cassette expression vectors. (A) Average IgA yield of three biological replicates upon expression of IgA1, IgA2m1, and IgA2m2 using multi-cassette vectors harvested 6 dpi. IgA yield in leaf extracts was determined by sandwich ELISA using a kappa chain specific capture and alpha heavy chain specific detection antibody. Error bars indicate standard error. (B) Average slgA yield of three biological replicates upon expression of slgA1, slgA2m1, and slgA2m2 using multi-cassette vectors (vector system 1 in Figure 3B) and harvested 6 dpi. slgA yield in leaf extracts was determined by sandwich ELISA using a kappa chain specific capture and secretory component specific detection antibody. Error bars indicate standard error.

chain expression using the 3-vector system. sIgA yield was determined by sandwich ELISA and joining chain expression was evaluated using western blot analysis (Figures 7A,B). While the expression of the joining chain increased by using a higher OD of the Agrobacterium culture harboring the vector for the joining chain expression, sIgA yield did not increase. We therefore assume that not joining chain expression, but its incorporation in the dIgA complex is the limiting factor for sIgA yield.

On a side note, the band that most likely represents monomeric joining chain $(\sim 25 \mathrm{kDa})$ appears to be a doublet (two bands migrating very close to each other). A doublet may represent the same protein with a different number of $N$-glycans. Because the joining chain harbors only one $N$-glycosylation site, this doublet should represent non-glycosylated and glycosylated joining chain.

To evaluate if lowering IgA expression would influence the IgA:sIgA ratio, we also attempted to decrease the amount of $\operatorname{IgA}$ by reducing the OD of the Agrobacterium culture harboring the vector for IgA expression using the 3-vector system. Again IIgA yield was determined by sandwich ELISA and sIgA assembly
A

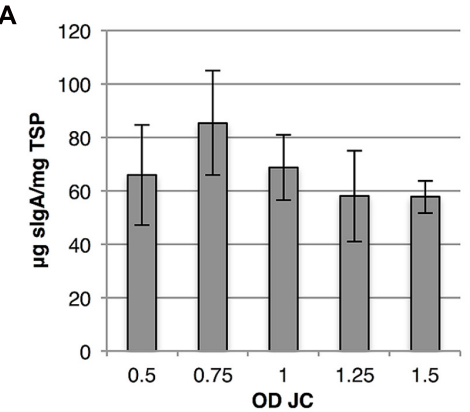

C

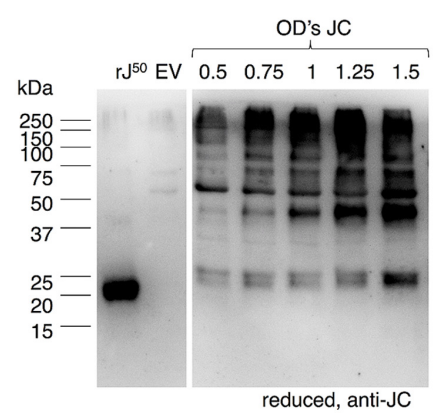

B

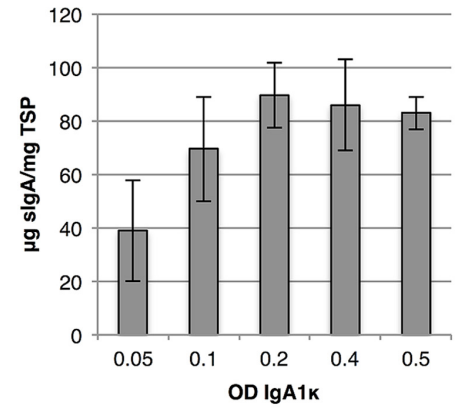

D

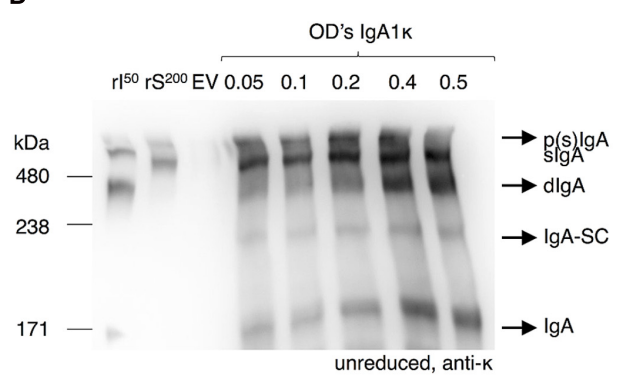

FIGURE 7 | Incorporation of the joining chain is the limiting factor for slgA yield. Leaves were agro-infiltrated for the expression of IgA1, JC and SC by co-infiltration (vector system 3 in Figure 3B) and harvested 6 dpi. (A) Average slgA yield ( $n=3$, error bars indicate standard error) and (B) western blot analysis upon increasing the OD of the Agrobacterium culture facilitating joining chain expression. As controls recombinant joining chain $\left(\mathrm{r} \mathrm{J}^{50}\right)$ and an $\mathrm{EV}$ sample were used. (C) Average slgA yield of three biological replicates and (D) western blot analysis of slgA assembly upon decreasing OD of the Agrobacterium culture facilitating IgA expression. As controls $50 \mathrm{ng}$ of recombinant $\lg \mathrm{A} 1 \mathrm{\kappa}\left(\mathrm{r}^{50}\right)$ and $200 \mathrm{ng}$ purified $\operatorname{slgA}\left(\mathrm{rS}^{200}\right)$ and an EV sample were used. 
was evaluated using western blot analysis (Figures 7C,D). Upon reduction of the OD of the Agrobacterium culture harboring the vector for IgA expression the amount of monomeric IgA reduces, but does not disappear. Also sIgA yield is reduced when the OD of the Agrobacterium culture that harbors the vector for IgA expression becomes lower than 0.2. Thus, the IgA:sIgA ratio does not change by lowering the expression of IgA. In other words, sIgA assembly cannot be improved by adjusting the expression of its individual components. It is therefore likely that not the capacity of the plant cell to assemble the protein complex, but intrinsic properties of the individual proteins determine $\operatorname{dIgA}$ assembly efficiency.

Moreover we also observed a significant proportion of $\operatorname{dIgA}$ when using ODs of 0.4 and 0.5 for IgA expression. In the previous results section we concluded that secretory component expression and association with $\operatorname{dIgA}$ was not limiting, as we hardly observed dIgA for any of the sIgA variants upon secretory component co-expression (Figure 5). However, the experiment described in the previous section was performed with the 1vector system and the experiment described in this section was performed with the 3 -vector system. This suggests that a significant proportion of cells does not receive the secretory component expression cassette upon co-infiltration or that at least the expression of the secretory component may vary from cell to cell.

\section{DISCUSSION}

We have studied the plant-based expression and assembly of three $\operatorname{sg} \mathrm{A}$ variants of the clinical antibody Ustekinumab (CNTO1275). We focussed on transient expression in $N$. benthamiana, as transient expression often yields more protein compared to stable transformation. Because $\operatorname{sigA}$ is a heteromultimeric protein complex transient expression can be achieved in several ways. Agrobacterium cultures each harboring expression vectors that facilitate expression of the individual components can be co-infiltrated or a multi-cassette expression vector facilitating expression of the four components can be used. Also, a combination of these two strategies may be adopted. The risk with co-infiltration is that perhaps a proportion of the plant cells will not be transformed with all genes. This may result in the presence of un-associated components or assembly intermediates that may complicate downstream processing. Use of a multi-cassette vector would ensure that each transformed cell expresses each gene, however, the much larger T-DNA may be less efficiently transferred into the plant cells.

We co-infiltrated all genes individually (4-vector system), coinfiltrated the secretory component and joining chain with the multi-cassette vector for IgA1א expression (3-vector system), co-infiltrated the secretory component and the multi-cassette

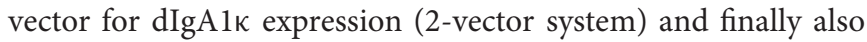
used a single multi-cassette vector for sIgAl $\kappa$ expression (1vector system). While sIgA yield was similar between the 4-, 3 -, and 2-vector systems, surprisingly the use of the 1-vector system increased yield twofold. This yield increase may be explained by an increased number of cells that receive and express all genes. This hypothesis is supported by the fact that the presence of dimeric IgA was more dominant using the 3-vector system compared to the 1-vector system. This suggests that the secretory component is not expressed in all cells when using a separate vector for its expression. However, when transformation efficiency is a yield-limiting factor for sIgA, a yield increase would be expected every time the number of expression vectors is reduced. As mentioned, the 4-, 3-, and 2-vector systems yielded a similar amount of sIgA. Perhaps the T-DNA containing the gene for the secretory component is not efficiently transferred to plant cells, while all others reach most cells even when separate vectors are used. The T-DNA containing the secretory component gene is the largest of the four (joining chain $4.6 \mathrm{kbp}$, kappa chain $4.8 \mathrm{kbp}$, alpha heavy chain $5.6 \mathrm{kbp}$ and the secretory component $5.9 \mathrm{kbp}$ ) and transformation efficiency has been demonstrated to go down with increased insert size (Frary and Hamilton, 2001). When the size of a T-DNA decreases transformation efficiency it would also reduce the efficiency by which the much larger T-DNA's of the multi-cassette vectors are transferred into plant cells (IgA $7.7 \mathrm{kpb}$, dIgA $9.6 \mathrm{kpb}$ and sIgA $12.8 \mathrm{kpb}$ ). This means that the yield increase due to the fact that more cells receive all genes every time the number of expression vectors is reduced, is compensated by a reduced number of transformed cells. Only in the case of the 1vectory system the yield increase is higher than the yield loss due to lower transformation efficiency because each transformed cell expresses all genes.

Next to ensuring that all genes are transferred to each transformed plant cell, the 1-vector system has another benefit. The 1-vector system also enables an increased copy number of each expression cassette. With the 4-vector system each Agrobacterium culture was used with an OD of 0.5 giving the final infiltration mix an OD of 2.5 (also including an Agrobacterium culture for expression of the viral silencing inhibitor p19 with an OD of 0.5 ), which we consider a maximum OD to allow efficient infiltration. With the 1-vector system an OD of 2.0 can be used until this maximum is reached. This means that the presence of each expression cassette is increased fourfold. While this may not results in a fourfold increase of each expression cassette in the plant cells due to a lower transformation efficiency of larger T-DNAs, it did increase sIgA yield 1.6-fold.

While the 1-vector system facilitated the highest yield of our protein complex, co-infiltration may still yield more protein complex if the expression of the individual genes does not reflect the stoichiometric ratio of the protein complex. If so, the expression of individual genes may be adjusted by controlling the concentration of the Agrobacterium cultures to best reflect the stoichiometric ratio of the protein complex. If free polypeptides are found, it can never be concluded whether or not these arise from unbalanced expression or are a consequence of partial transformation. Alternatively, use of promoters with different strengths could be used that enable the accumulation of the individual proteins in the stoichiometric ratio of sIgA and allow the use of a 1-vector system. While testing of different promoters may be very laborious, systems are arising that allow easy high throughput cloning, such as the golden gate system (Engler et al., 2009). 
Even when using a single multi-cassette expression vector, accumulation of assembly intermediates still occurred, with monomeric IgA as the most predominant intermediate. This is in line with four other studies on expression of murine, chicken and human sIgA in plants that all report the accumulation of a significant proportion of monomeric IgA next to $\operatorname{sg} A$ (Ma et al., 1995; Wieland et al., 2006; Juarez et al., 2013; Paul et al., 2014). Follow up studies on the expression of murine (s)IgA demonstrated that this antibody was targeted to the vacuole due to a cryptic targeting signal in the tailpiece of murine IgA (Frigerio et al., 2000; Hadlington et al., 2003). In our previous publication on expression of monomeric human IgA we demonstrate that also human IgA is poorly secreted from plant cells (Westerhof et al., 2014). The tailpieces of both human and chicken IgA contain similar sequences as the suggested cryptic targeting signal of murine IgA. Thus, it may well be that also chicken and human IgA are targeted to the vacuole. If $\operatorname{IgA}$ is targeted to the vacuole, it is possible that a proportion of $\operatorname{IgA}$ is transported to the vacuole before the joining chain can be incorporated. Unfortunately the tailpiece of IgA cannot be removed, as the penultimate cysteine residue forms a disulphide bond with a free cysteine of the joining chain (Atkin et al., 1996). An investigation whether mutations in the cryptic vacuolar targeting signal can abolish vacuolar targeting without influencing the complex assembly may provide a solution.

Reports on the expression of sIgA in Chinese hamster ovary (CHO) cells also identify dIgA assembly as the yield-limiting step in sIgA expression (Berdoz et al., 1999; Li et al., 2014). Because mammalian cells are devoid of vacuoles, vacuolar targeting cannot explain the lack of sIgA assembly. Unfortunately, both studies did not determine joining chain expression. Thus it is unclear if sIgA assembly in CHO cells is caused by limited joining chain expression or inefficient incorporation of the joining chain. To evaluate if either joining chain expression or incorporation was the limiting step for the yield of our sIgA we increased the bacterial OD of the Agrobacterium culture. Increasing the Agrobacterium concentration facilitated increased joining chain expression. Even though joining chain expression was increased, sIgA yield was not, nor had the proportion of monomeric IgA diminished. We therefore assume that joining chain incorporation was the limiting step for sIgA assembly.

We also observed that the band assumed to represent monomeric joining chain migrated as a doublet. Because the joining chain harbors only one $N$-glycosylation site it is possible that this doublet represents a non-glycosylated and a glycosylated version of the joining chain. It was demonstrated that incorporation of the joining chain is reduced if asparagine 48 of the joining chain or asparagine 549 of the alpha heavy chain is not glycosylated (Atkin et al., 1996; Krugmann et al., 1997). In a previous study, we already confirmed that the $N$-glycosylation of the asparagine 549 of the alpha heavy chain is partial (Westerhof et al., 2014). Partial N-glycosylation as a reason for inefficient incorporation of the joining chain coincides with the fact that also reduced expression of $\operatorname{IgA}$ did not alter the IgA:sIgA ratio. We therefore hypothesize that the capacity of the plant cell to assemble $\operatorname{dIgA}$ is not limiting. Partial $N$-glycosylation of IgA and/or joining chain explains inefficient sIgA assembly both for plant as well as $\mathrm{CHO}$ cell produced sIgA. Because $N$-glycosylation is co-translational limited access to the $N$-glycosylation site cannot explain inefficient $N$-glycosylation. However, in a largescale analysis of glycoproteins it was suggested that the sequence surrounding an $\mathrm{N}$-glycosylation signal may influence $N$-glycosylation efficiency (Petrescu et al., 2004). Perhaps adaptation of the sequences surrounding the $N$-glycosylation sites in the joining chain and tailpiece of the alpha heavy chain can increase $N$-glycosylation efficiency. If this would increase sIgA assembly and reduce the presence of sIgA intermediates it would not only increase yield, but also simplify purification procedures.

Taken together our data suggests that plants most certainly allow the economic production of heteromultimeric protein complexes such as sIgA. The maximum yield of our sIgA1к-Ustekinumab variant was $37 \%$ of TSP, which is well above the $1 \%$ commercial viability threshold. Hereby transient expression with use of a multi-cassette expression vector is the best strategy, because it ensures expression of all genes in all transformed cells. This prevents the occurrence of assembly intermediates due to partial transformation.

\section{AUTHOR CONTRIBUTIONS}

LW - Has had the lead in this research project (concept and design), acquisition of data, analysis and interpretation of data, drafting and revising the article, and final approval of the version to be published.

RW - Substantial contributions to concept and design, acquisition of data, analysis and interpretation of data, and writing and revising the article critically for important intellectual content and final approval of the version to be published.

DvR - Acquisition of data.

CvW - Acquisition of data.

AG - Writing and revising the article critically for important intellectual content.

JB - Writing and revising the article critically for important intellectual content.

AS - Writing and revising the article critically for important intellectual content and final approval of the version to be published.

\section{FUNDING}

This research was financially supported in part by Synthon (Nijmegen, The Netherlands) and a grant from the Dutch Ministry of Economic Affairs (PID07124).

\section{ACKNOWLEDGMENTS}

We would like to thank Tim Warbroek, Aleksandra Syta, and Bob Engelen for their input in the experimental work and Gerard Rouwendal for his help in designing the multi-cassette vector system. 


\section{REFERENCES}

Atkin, J. D., Pleass, R. J., Owens, R. J., and Woof, J. M. (1996). Mutagenesis of the human IgA1 heavy chain tailpiece that prevents dimer assembly. J. Immunol. 157, 156-159.

Berdoz, J., Blanc, C. T., Reinhardt, M., Kraehenbuhl, J. P., and Corthesy, B. (1999). In vitro comparison of the antigen-binding and stability properties of the various molecular forms of $\operatorname{IgA}$ antibodies assembled and produced in $\mathrm{CHO}$ cells. Proc. Natl. Acad. Sci. U.S.A. 96, 3029-3034. doi: 10.1073/Pnas.96.6.3029

Bhol, K. C., Tracey, D. E., Lemos, B. R., Lyng, G. D., Erlich, E. C., Keane, D. M., et al. (2013). AVX-470: a novel oral anti-TNF antibody with therapeutic potential in inflammatory bowel disease. Inflamm. Bowel Dis. 19, 2273-2281. doi: 10.1097/MIB.0b013e3182a11958

Bosch, D., Castilho, A., Loos, A., Schots, A., and Steinkellner, H. (2013). N-Glycosylation of plant-produced recombinant proteins. Curr. Pharm. Des. 19, 5503-5512. doi: 10.2174/1381612811319310006

Castilho, A., Neumann, L., Daskalova, S., Mason, H. S., Steinkellner, H., Altmann, F., et al. (2012). Engineering of sialylated mucin-type O-glycosylation in plants. J. Biol. Chem. 287, 36518-36526. doi: 10.1074/jbc.M112.402685

Chen, Q., and Lai, H. (2013). Plant-derived virus-like particles as vaccines. Hum. Vaccin. Immunother. 9, 26-49. doi: 10.4161/hv.22218

Conley, M. E., and Delacroix, D. L. (1987). Intravascular and mucosal immunoglobulin a: two separate but related systems of immune defense? Ann. Intern. Med. 106, 892-899. doi: 10.7326/0003-4819-106-6-892

De Muynck, B., Navarre, C., and Boutry, M. (2010). Production of antibodies in plants: status after twenty years. Plant Biotechnol. J. 8, 529-563. doi: 10.1111/j.1467-7652.2009.00494.x

Delacroix, D. L., Dive, C., Rambaud, J. C., and Vaerman, J. P. (1982). IgA subclasses in various secretions and in serum. Immunology 47, 383-385.

Engler, C., Gruetzner, R., Kandzia, R., and Marillonnet, S. (2009). Golden gate shuffling: a one-pot DNA shuffling method based on type IIs restriction enzymes. PLoS ONE 4:e5553. doi: 10.1371/journal.pone.0005553

Enriquez, F. J., and Riggs, M. W. (1998). Role of immunoglobulin A monoclonal antibodies against P23 in controlling murine Cryptosporidium parvum infection. Infect. Immun. 66, 4469-4473.

Frary, A., and Hamilton, C. M. (2001). Efficiency and stability of high molecular weight DNA transformation: an analysis in tomato. Transgenic Res. 10, 121132. doi: 10.1023/A:1008924726270

Frigerio, L., Vine, N. D., Pedrazzini, E., Hein, M. B., Wang, F., Ma, J. K. C., et al. (2000). Assembly, secretion, and vacuolar delivery of a hybrid immunoglobulin in plants. Plant Physiol. 123, 1483-1493. doi: 10.1104/Pp.123.4.1483

Hadlington, J. L., Santoro, A., Nuttall, J., Denecke, J., Ma, J. K. C., Vitale, A., et al. (2003). The C-terminal extension of a hybrid immunoglobulin $\mathrm{A} / \mathrm{G}$ heavy chain is responsible for its golgi-mediated sorting to the vacuole. Mol. Biol. Cell 14, 2592-2602. doi: 10.1091/Mbc.E02-11-0771

Juarez, P., Huet-Trujillo, E., Sarrion-Perdigones, A., Falconi, E. E., Granell, A., and Orzaez, D. (2013). Combinatorial analysis of secretory immunoglobulin A (sIgA) expression in plants. Int. J. Mol. Sci. 14, 6205-6222. doi: 10.3390/ijms14036205

Keane, J., Gershon, S., Wise, R. P., Mirabile-Levens, E., Kasznica, J., Schwieterman, W. D., et al. (2001). Tuberculosis associated with infliximab, a tumor necrosis factor (alpha)-neutralizing agent. N. Engl. J. Med. 345, 1098-1104. doi: 10.1056/Nejmoa011110

Kerr, A. (1990). The structure and function of human IgA. Biochem. J. 271, 285-296. doi: 10.1042/bj2710285

Krugmann, S., Pleass, R. J., Atkin, J. D., and Woof, J. M. (1997). Structural requirements for assembly of dimeric IgA probed by site-directed mutagenesis of J chain and a cysteine residue of the alpha-chain $\mathrm{CH} 2$ domain. J. Immunol. 159, 244-249.

Li, C., An, X. P., Butt, A. M., Zhang, B. Z., Zhang, Z. Y., Wang, X. N., et al. (2014). Construction of a chimeric secretory IgA and Its neutralization activity against avian influenza virus H5N1. J. Immunol. Res. 2014, 394127. doi: $10.1155 / 2014 / 394127$

Ma, J. K., Hiatt, A., Hein, M., Vine, N. D., Wang, F., Stabila, P., et al. (1995). Generation and assembly of secretory antibodies in plants. Science 268, 716719. doi: $10.1126 /$ science. 7732380

Ma, J. K., Hikmat, B. Y., Wycoff, K., Vine, N. D., Chargelegue, D., Yu, L., et al. (1998). Characterization of a recombinant plant monoclonal secretory antibody and preventive immunotherapy in humans. Nat. Med. 4, 601-606. doi: 10.1038/nm0598-601

Narimatsu, Y., Kubota, T., Furukawa, S., Morii, H., Narimatsu, H., and Yamasaki, K. (2010). Effect of glycosylation on cis/trans isomerization of prolines in IgA1-hinge peptide. J. Am. Chem. Soc. 132, 5548-5549. doi: $10.1021 /$ ja9106429

Neurath, M. F. (2014). Cytokines in inflammatory bowel disease. Nat. Rev. Immunol. 14, 329-342. doi: 10.1038/nri3661

Paul, M., Reljic, R., Klein, K., Drake, P. M., Van Dolleweerd, C., Pabst, M., et al. (2014). Characterization of a plant-produced recombinant human secretory IgA with broad neutralizing activity against HIV. MAbs 6, 1585-1597. doi: 10.4161/mabs.36336

Petrescu, A. J., Milac, A. L., Petrescu, S. M., Dwek, R. A., and Wormald, M. R. (2004). Statistical analysis of the protein environment of N-glycosylation sites: implications for occupancy, structure, and folding. Glycobiology 14, 103-114. doi: 10.1093/Glycob/Cwh008

Royle, L., Roos, A., Harvey, D. J., Wormald, M. R., Van Gijlswijk-Janssen, D., Redwan, E. M., et al. (2003). Secretory IgA N- and O-glycans provide a link between the innate and adaptive immune systems. J. Biol. Chem. 278, 20140-20153. doi: 10.1074/Jbc.M301436200

Stoop, J. W., Zegers, B. J., Sander, P. C., and Ballieux, R. E. (1969). Serum immunoglobulin levels in healthy children and adults. Clin. Exp. Immunol. 4, 101-112.

Vaerman, J.-P., Heremans, J. F., and Laurell, C. B. (1968). Distribution of alpha chain subclasses in normal and pathological IgA-globulins. Immunology 14, 425-432.

van Engelen, F. A., Schouten, A., Molthoff, J. W., Roosien, J., Salinas, J., Dirkse W. G., et al. (1994). Coordinate expression of antibody subunit genes yields high levels of functional antibodies in roots of transgenic tobacco. Plant Mol. Biol. 26, 1701-1710. doi: 10.1007/BF00019485

Voinnet, O., Rivas, S., Mestre, P., and Baulcombe, D. (2003). An enhanced transient expression system in plants based on suppression of gene silencing by the p19 protein of tomato bushy stunt virus. Plant J. 33, 949-956. doi: 10.1046/j.1365313X.2003.01676.x

Westerhof, L. B., Wilbers, R. H. P., Raaij Van, D. R., Nguyen, D.-L., Goverse, A., Henquet, M. G. L., et al. (2014). Monomeric IgA can be produced in planta as efficient as IgG, yet receives different N-glycans. Plant Biotechnol. J. 12, 1333-1342. doi: 10.1111/pbi.12251

Westerhof, L. B., Wilbers, R. H. P., Roosien, J., Van De Velde, J., Goverse, A., Bakker, J., et al. (2012). 3D domain swapping causes extensive multimerisation of human interleukin-10 when expressed in planta. PLOS ONE 7:e46460. doi: 10.1371/journal.pone.0046460

Wieland, W. H., Lammers, A., Schots, A., and Orzaez, D. V. (2006). Plant expression of chicken secretory antibodies derived from combinatorial libraries. J. Biotechnol. 122, 382-391. doi: 10.1016/j.jbiotec.2005.12.020

Williams, A., Reljic, R., Naylor, I., Clark, S. O., Falero-Diaz, G., Singh, M., et al. (2004). Passive protection with immunoglobulin A antibodies against tuberculous early infection of the lungs. Immunology 111, 328-333. doi: 10.1111/j.1365-2567.2004.01809.x

Yang, Z., Drew, D. P., Jorgensen, B., Mandel, U., Bach, S. S., Ulvskov, P., et al. (2012). Engineering mammalian mucin-type O-glycosylation in plants. J. Biol. Chem. 287, 11911-11923. doi: 10.1074/jbc.M111.312918

Conflict of Interest Statement: The authors declare that the research was conducted in the absence of any commercial or financial relationships that could be construed as a potential conflict of interest.

The reviewer Marcello Donini and handling Editor Eugenio Benvenuto declared their shared affiliation, and the handling Editor states that, nevertheless, the process met the standards of a fair and objective review.

Copyright (C) 2016 Westerhof, Wilbers, van Raaij, van Wijk, Goverse, Bakker and Schots. This is an open-access article distributed under the terms of the Creative Commons Attribution License (CC BY). The use, distribution or reproduction in other forums is permitted, provided the original author(s) or licensor are credited and that the original publication in this journal is cited, in accordance with accepted academic practice. No use, distribution or reproduction is permitted which does not comply with these terms. 